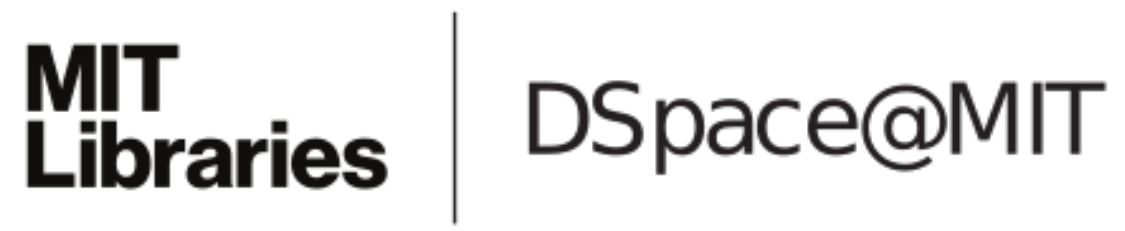

\author{
MIT Open Access Articles
}

Resonance-enhanced waveguidecoupled silicon-germanium detector

The MIT Faculty has made this article openly available. Please share how this access benefits you. Your story matters.

Citation: Alloatti, L. and Ram, R. J. “Resonance-Enhanced Waveguide-Coupled SiliconGermanium Detector." Applied Physics Letters 108, 7 (February 2016): 071105 (C) 2016 AIP Publishing LLC

As Published: http://dx.doi.org/10.1063/1.4941995

Publisher: American Institute of Physics (AIP)

Persistent URL: http://hdl.handle.net/1721.1/110934

Version: Final published version: final published article, as it appeared in a journal, conference proceedings, or other formally published context

Terms of Use: Article is made available in accordance with the publisher's policy and may be subject to US copyright law. Please refer to the publisher's site for terms of use. 


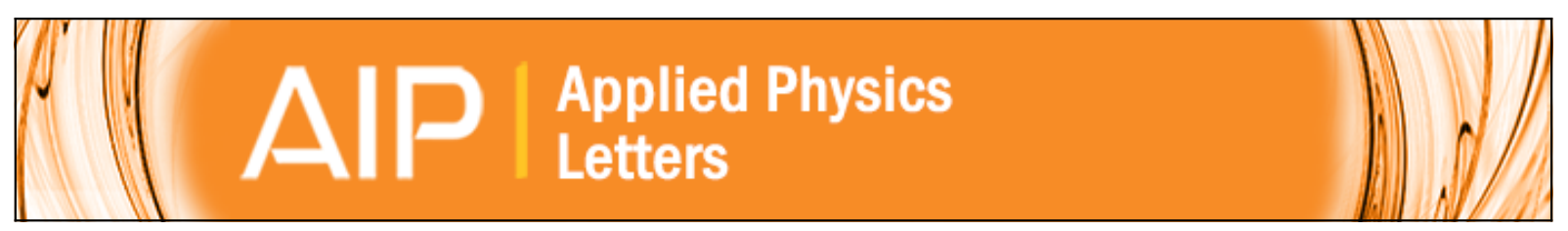

\section{Resonance-enhanced waveguide-coupled silicon-germanium detector}

L. Alloatti and R. J. Ram

Citation: Applied Physics Letters 108, 071105 (2016); doi: 10.1063/1.4941995

View online: http://dx.doi.org/10.1063/1.4941995

View Table of Contents: http://scitation.aip.org/content/aip/journal/apl/108/7?ver=pdfcov

Published by the AIP Publishing

\section{Articles you may be interested in}

Waveguide-coupled detector in zero-change complementary metal-oxide-semiconductor

Appl. Phys. Lett. 107, 041104 (2015); 10.1063/1.4927393

Efficient evanescent wave coupling conditions for waveguide-integrated thin-film Si/Ge photodetectors on siliconon-insulator/germanium-on-insulator substrates

J. Appl. Phys. 110, 083115 (2011); 10.1063/1.3642943

Metal-semiconductor-metal Ge photodetectors integrated in silicon waveguides

Appl. Phys. Lett. 92, 151114 (2008); 10.1063/1.2909590

Guided-wave near-infrared detector in polycrystalline germanium on silicon

Appl. Phys. Lett. 87, 203507 (2005); 10.1063/1.2131175

Back-side-illuminated high-speed Ge photodetector fabricated on Si substrate using thin SiGe buffer layers

Appl. Phys. Lett. 85, 3286 (2004); 10.1063/1.1805706

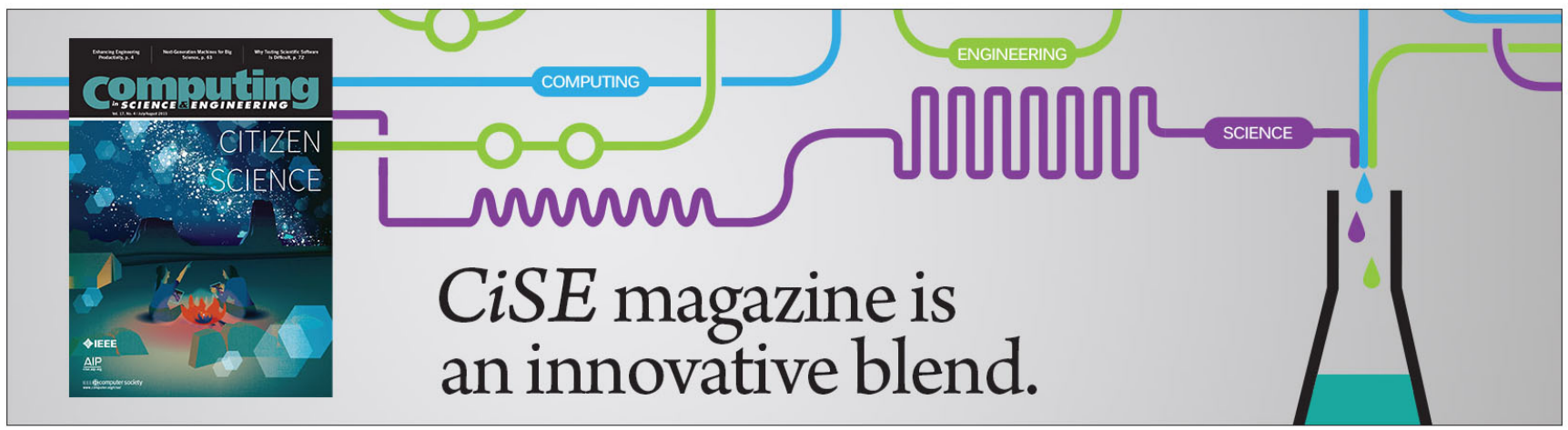




\title{
Resonance-enhanced waveguide-coupled silicon-germanium detector
}

\author{
L. Alloatti ${ }^{\text {a),b) }}$ and R. J. Ram \\ Massachusetts Institute of Technology, Cambridge, Massachusetts 02139, USA
}

(Received 5 January 2016; accepted 3 February 2016; published online 16 February 2016)

\begin{abstract}
A photodiode with $0.55 \pm 0.1 \mathrm{~A} / \mathrm{W}$ responsivity at a wavelength of $1176.9 \mathrm{~nm}$ has been fabricated in a $45 \mathrm{~nm}$ microelectronics silicon-on-insulator foundry process. The resonant waveguide photodetector exploits carrier generation in silicon-germanium within a microring which is compatible with high-performance electronics. A $3 \mathrm{~dB}$ bandwidth of $5 \mathrm{GHz}$ at $-4 \mathrm{~V}$ bias is obtained with a dark current of less than $20 \mathrm{pA}$. ( 2016 AIP Publishing LLC. [http://dx.doi.org/10.1063/1.4941995]
\end{abstract}

Single-chip microprocessors can exceed a compute capacity of $5 \times 10^{12}$ floating-point operations per second ( 5 TFLOPS) ${ }^{1}$ therefore, requiring an input/output (I/O) bandwidth of $40 \mathrm{~Tb} / \mathrm{s}$ consistent with the approximately "one byte $\mathrm{I} / \mathrm{O}$ per flop" rule-of-thumb. ${ }^{2,3}$ However, the physical limitations of electrical interconnects-which are constrained by RF losses, electromagnetic interference, power dissipation, and package pin density-typically limit the available bandwidth to a tenth of the peak bandwidth required.

Monolithic integration of optical transceivers side-byside with billion-transistor circuits has the potential to overcome these limitations. However, achieving the necessary transistors' yield together with high photonics performance has been a major challenge. Monolithic approaches developed so far have followed the path of modifying existing electronic processes by adding fabrication steps and materials such as pure germanium for photocarrier generation ${ }^{4,5}$ with the risk of shifting the transistor specifications and decreasing the fabrication yield. These processes moreover exploit $90 \mathrm{~nm}$ or older nodes which are not currently utilized for building high-performance computers (HPC) ${ }^{6,7}$

An alternative approach consists of designing photonic components in existing CMOS nodes without violating any design rule and without requiring any modifications to the process flow-the so-called zero-change CMOS. ${ }^{8,9}$ Within the GlobalFoundries (formerly IBM) $45 \mathrm{~nm}$ 12SOI node, we have recently demonstrated a complete zero-change photonic toolbox comprising waveguides with $5 \mathrm{~dB} / \mathrm{cm}$ propagation losses, ${ }^{8}$ grating-couplers, ${ }^{10} 5 \mathrm{Gbps}$ modulators, ${ }^{11}$ and $32 \mathrm{GHz}$ photodetectors. ${ }^{7}$ These components enabled the first realization of an optical link between a microprocessor and an external memory. ${ }^{12}$ However, the responsivity of the first photodiodes was limited to $\sim 0.02 \mathrm{~A} / \mathrm{W}$ and directly impacted the power efficiency of the link. ${ }^{12}$

In this work, we present a zero-change CMOS photodiode with a responsivity of $0.55 \pm 0.1 \mathrm{~A} / \mathrm{W}-\mathrm{a}$ twenty-fold increase over previous results. ${ }^{12}$ Similar to our first demonstration, the photodiode exploits carrier generation in silicon-germanium ( $\mathrm{SiGe}$ ) which is already present in the $45 \mathrm{~nm}$ 12SOI node for stressing the channels of p-FETs. ${ }^{7}$ To increase the responsivity, here we exploit resonance-

\footnotetext{
${ }^{a)}$ Current address: Institute of Electromagnetic Fields (IEF), ETH Zurich, Zurich, Switzerland.

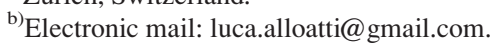

enhancement in a microring. The disk-like cavity supports a whispering gallery mode for effectively separating the optical field from the metal contacts. ${ }^{11}$ The cavity has a radius of $5 \mu \mathrm{m}$ and is etched into the crystalline silicon which is normally used in the $45 \mathrm{~nm}$ process for realizing the body of the transistors. ${ }^{8}$ This type of cavity has already been exploited for building modulators, ${ }^{11}$ but the gap between the bus waveguide and the ring has been reduced for compensating the higher absorption of the detector, low-resistance contacts have been added between silicon and vias, and the number of pn-junctions has been reduced to minimize the device resistance and capacitance. A closed-loop circuit and a thermal heater can effectively lock the resonator to the desired wavelength as demonstrated elsewhere on the chip. ${ }^{13}$ Inside this cavity, a $500 \mathrm{~nm}$ wide ring is partially etched into the silicon for forming the heteroepitaxially-grown $\mathrm{SiGe}$ absorption region, Fig. 1. The SiGe alloy has a germanium content between $25 \%$ and $35 \%$ based on literature data and on previous experiments. ${ }^{7}$ Two n-type and two p-type spoke-shaped well-implants form interdigitated pn-junctions in the SiGe region and connect the active region to the contacts located on the inner radius, Fig. 1(b). The p-type and n-type spokes have different lengths so that a part of the pn-junction is located in the middle of the SiGe section. These wellimplants are performed prior to SiGe deposition and affect the silicon only. It is not known whether the SiGe has been doped chemically during the deposition, and it is possible that it is nearly intrinsic. The implant spokes are narrower in the inner part of the cavity (T-shapes) for reducing the parasitic junction capacitance between the SiGe region and the electrical contacts. Source-drain (S/D) well implants, halo/ extension implants, and silicidation complete the electrical contact to the high-frequency ground-signal (GS) electrodes. ${ }^{7}$

To facilitate testing, broadband grating couplers $(1170 \mathrm{~nm}-1560 \mathrm{~nm})$ are used to couple light in and out of the waveguide and cause a wavelength-dependent loss of at least $10 \mathrm{~dB}$ each although optimized grating couplers have been demonstrated elsewhere on this chip with $1.2 \mathrm{~dB}$ insertion loss. ${ }^{10}$ The waveguides have been designed by a fully scripted photonic-design automation (PDA) tool based on Cadence with automatic DRC-cleaning and layer generation. ${ }^{9}$ The present device belongs to a sweep of 60 variations obtained by permuting 5 different coupling gaps, 6 doping patterns, and $2 \mathrm{SiGe}$ ring widths. The chip was taped-out in March 2015, and the manufacturing was completed by 
September 2015. Among the variations on the doping patterns, the present device shows the highest bandwidth. Other variations contain $\mathrm{T}$-shaped spokes having equal dimensions for both the n-implants and p-implants extending from the inner radius to the outer radius of the cavity so that the junctions cut the SiGe region radially. Neither halo nor extension implants (which would affect the SiGe as well) have been used on the optical cavities yet, and they may be used to improve the junction doping profile in future generations.

The current-voltage characteristic is shown under different illumination conditions in Fig. 1(a). The dark current is smaller than $20 \mathrm{pA}$ in the reverse bias range $-5 \mathrm{~V}$ to $0 \mathrm{~V}$ corresponding to a dark current density of $0.116 \mathrm{~mA} / \mathrm{cm}^{2}$. This value is significantly smaller than for the majority of germanium-based photodiodes demonstrated so far in silicon photonics ${ }^{14-17}$ and is close to the thermionic emission limit of germanium photodiodes which is $\sim 10^{-2} \mathrm{~mA} / \mathrm{cm}^{2}$. ${ }^{17}$ The small dark current is attributed to a low density of defects and dislocations.

The responsivity vs. wavelength is shown in Fig. 2(b) and reaches a peak value of $0.55 \pm 0.1 \mathrm{~A} / \mathrm{W}$ at $\sim 1176.9 \mathrm{~nm}$

(a)
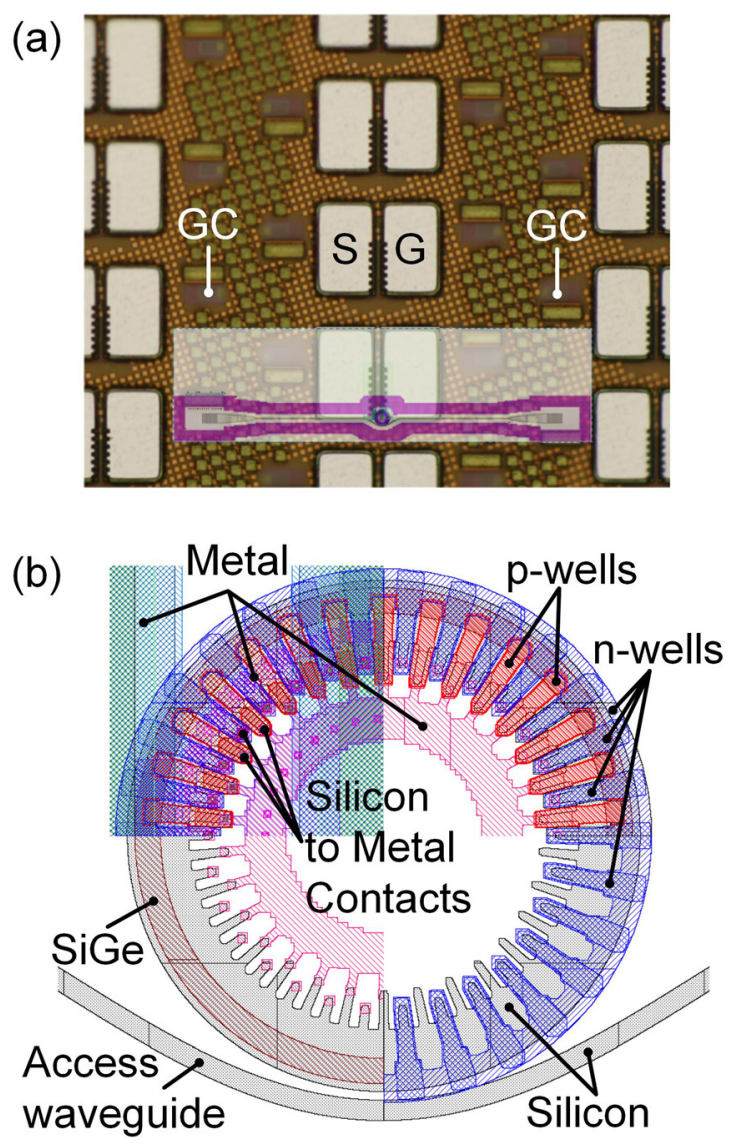

FIG. 1. Photodetector geometry. (a) Microphotograph of the fabricated chip showing a sweep of devices. The GS electrodes can be easily recognized along with the grating-couplers (GC) apertures through the metal stack. The layout of a single device is superimposed. (b) Layout of the cavity with the access waveguide. The cavity region is divided in four quadrants with different mask layers highlighted/hidden. In the top-left quadrant, most of the layers are activated including the high-doping regions (dark red and dark blue), the inner and outer metal contacts, and some vias. The top-right quadrant highlights the relative positions of the n-type and p-type well implants. In the bottom-left quadrant, only the $500 \mathrm{~nm}$ wide SiGe ring is visible together with the silicon cavity and one metal layer. In the bottom-right quadrant, only the n-implants and the silicon cavity are highlighted. The T shape of the implants is recognizable. corresponding to a quantum efficiency of $58 \%$. The data have been fitted with the model of a ring resonator having a FWHM transmission $\delta \lambda_{\text {FWHM }}=0.138 \mathrm{~nm}(29.9 \mathrm{GHz})$ corresponding to a loaded $\mathrm{Q}=\lambda / \delta \lambda_{\mathrm{FWHM}}=8530$. The responsivity was measured by recording photocurrent and optical transmission at the same time. The current was measured at low optical powers to minimize the drift of the resonant frequency by self-heating, ${ }^{13,18}$ and the insertion loss of the input and output grating couplers was subtracted out. The measurement was repeated for the second time with exchanged input and output fiber connectors such as to verify that the input and output grating couplers caused the same optical loss. ${ }^{7}$ The free spectral range (FSR) is $13.75 \mathrm{~nm}$, and the transmission at resonance is about $-15 \mathrm{~dB}$. The excitation of a higher order mode causes an additional responsivity peak red-shifted by $\sim 5 \mathrm{~nm}$ from the main resonance and suppressed by about $19 \mathrm{~dB}$.

The bandwidth of the device was measured by contacting the GS electrodes with a $50 \mu \mathrm{m}$ pitch GS probe of Cascade Microtech (model Infinity I67-A-GS-50). The reference plane was set at the $\mathrm{V}$-connector of the probe. The frequency response was measured with a $40 \mathrm{GHz}$ VNA (HP8722D), and the frequency-response of the setup (comprising modulator, RF cables, and bias-T) was calibrated with a reference photodiode (Discovery Semiconductors, model DSC30-3-2010) of known frequency-response. ${ }^{7}$ The frequency response was recorded for different bias voltages and different offsets from the resonant wavelength, therefore, resulting in different average currents (the in-waveguide power was kept constant), Fig. 2. For $0 \mathrm{~V}$ bias, the frequency response is almost independent of the optical power in the waveguide and follows a nearly exponential roll-off. ${ }^{19}$ For $-4 \mathrm{~V}$ bias, we observe that higher in-cavity optical powers (larger average currents) correspond to higher bandwidths. For both bias voltages, the frequency roll-off suggests that the device is not limited by the RC time constant. For $-4 \mathrm{~V}$ bias, the $3 \mathrm{~dB}$ bandwidth varies between $\sim 2 \mathrm{GHz}$ and $5 \mathrm{GHz}$ when the average current changes between $10 \mu \mathrm{A}$ and $100 \mu \mathrm{A}$. Since the frequency response was found earlier to be very sensitive to the position of the pn-junction relatively to the SiGe region, ${ }^{7}$ we expect that faster devices can be obtained by optimizing the doping profile. Eye diagrams have been recorded at $5 \mathrm{~Gb} / \mathrm{s}$ and $12.5 \mathrm{~Gb} / \mathrm{s}$ (PRBS length $2^{31}-1$ ) with an Agilent waveform analyzer (model 86108A with $50 \Omega$ termination), Fig. 3. In these experiments, neither optical nor electrical amplifiers have been used. Open eyes are obtained at $5 \mathrm{~Gb} / \mathrm{s}$ with $-1 \mathrm{~V}$ bias and at $12.5 \mathrm{~Gb} / \mathrm{s}$ at $-4 \mathrm{~V}$ bias with $120 \mu \mathrm{A}$ average current.

In conclusion, we have demonstrated a photodiode with a responsivity of $0.55 \pm 0.1 \mathrm{~A} / \mathrm{W}$ at $0 \mathrm{~V}$ bias in zero-change CMOS. The photodiode is realized in the $45 \mathrm{~nm} 12 \mathrm{SOI}$ CMOS node, which is widely used in HPC. The silicongermanium used for optical absorption is grown heteroepitaxially in circular silicon pockets and has a low germanium content in contrast to mainstream silicon-photonic photodiodes. We are not aware of previous demonstrations of germanium or silicon-germanium having boundaries which are not aligned with a major crystalline axis. High-responsivity photodiodes are key components for minimizing the optical power budget in future chip-to-chip transceivers. ${ }^{12}$ This detector, when 
(a)

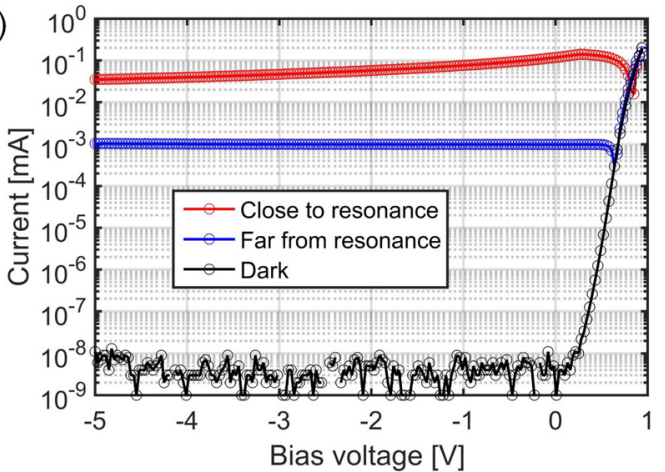

(b)

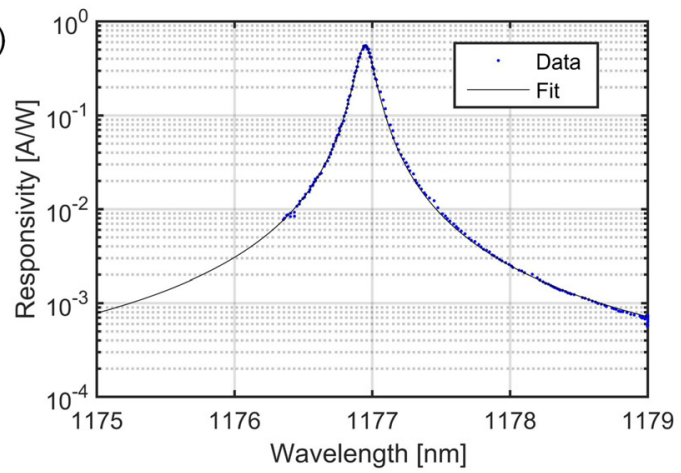

(c)

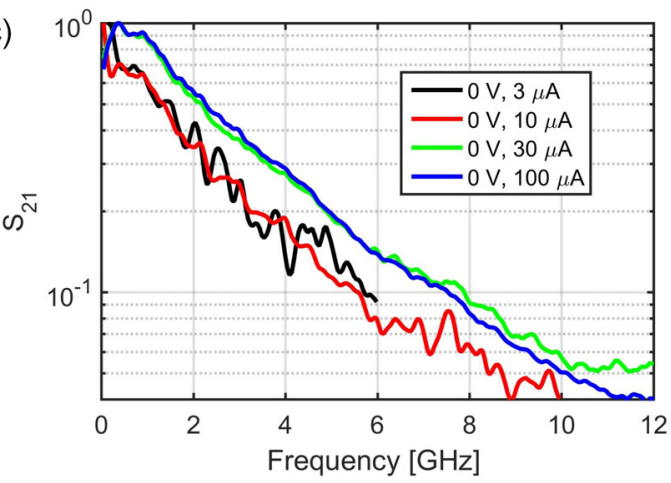

(d)

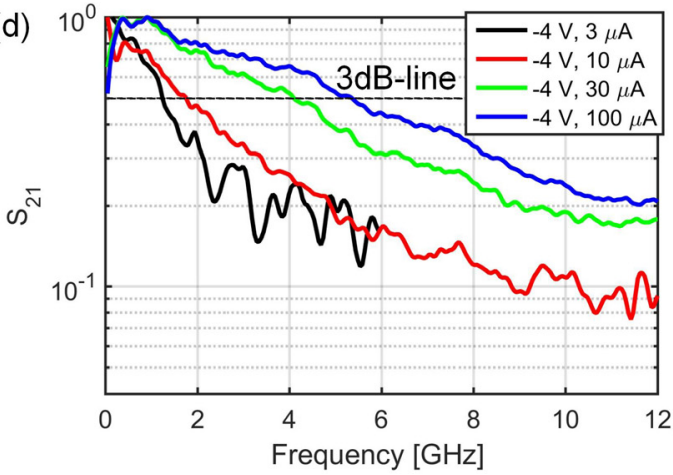

FIG. 2. Device performance. (a) Current-voltage characteristics for different illumination conditions. The dark current is smaller than $20 \mathrm{pA}$ for $-5 \mathrm{~V}$ to $0 \mathrm{~V}$ bias. When the wavelength is far from resonance, the reverse-bias photocurrent is approximately constant. The variation of the photocurrent when the wavelength is close to resonance is attributed to self-heating effects which detune the cavity. (b) Responsivity vs. wavelength. A maximum of $0.55 \mathrm{~A} / \mathrm{W}$ is observed. Data were recorded at low optical powers first by increasing the wavelength and then by decreasing it for excluding heating-induced drifts. The thermally tuned laser source (QDLaser model QLD1161-8030) was limited to wavelengths larger than $\sim 1176.4 \mathrm{~nm}$. (c) Frequency response at $0 \mathrm{~V}$ bias for different in-cavity optical powers (different average currents). In the low-frequency limit, the VNA measurements were not reproducible so that a $30 \%$ error should be taken into account on the normalization. (d) Frequency response at $-4 \mathrm{~V}$ bias for different average currents. A $3 \mathrm{~dB}$ bandwidth of $5 \mathrm{GHz}$ is obtained at $-4 \mathrm{~V}$ bias for an average current of $100 \mu \mathrm{A}$.

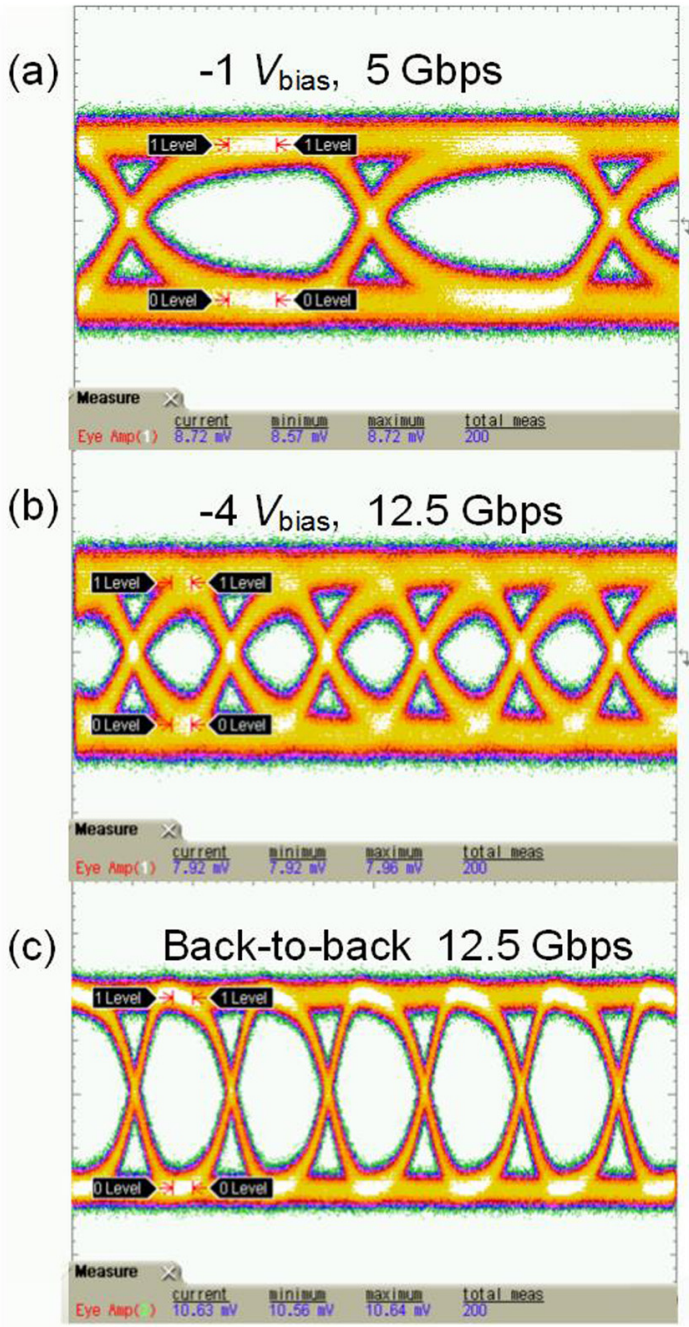

FIG. 3. Eye diagrams. (a) $5 \mathrm{~Gb} / \mathrm{s}$ and $-1 \mathrm{~V}$ bias. (b) $12.5 \mathrm{~Gb} / \mathrm{s}$ and $-4 \mathrm{~V}$ bias. (c) Back-to-back reference at $12.5 \mathrm{~Gb} / \mathrm{s}$ taken with a $30 \mathrm{GHz}$ commercial photodiode. All the eye diagrams have a vertical scale of $3 \mathrm{mV} / \mathrm{div}$. The same number of measurements was taken for all the eye diagrams.

coupled with previously demonstrated receivers having an average sensitivity of less than $3 \mu \mathrm{A}$ at $2.5 \mathrm{~Gb} / \mathrm{s},{ }^{13}$ would correspond to a sensitivity of $-22.6 \mathrm{dBm}, 17 \mathrm{~dB}$ improvement over previous demonstrations. ${ }^{12}$ The resonant design enables the detection of wavelength-division multiplexing (WDM) signals without introducing additional filters.

We acknowledge support by DARPA POEM under Award No. HR0011-11-C-0100 and Contract No. HR001111-9-0009. The views expressed are those of the authors and do not reflect the official policy or position of the DoD or the U.S. Government. We thank Amir Atabaki for performing the chip substrate transfer.

${ }^{1}$ W. Liu and B. Vinter, J. Parallel Distrib. Comput. 85, 47 (2015).

${ }^{2}$ J. S. Orcutt, R. J. Ram, and V. Stojanovic, "Optical fiber telecommunications volume VIA," Components and Subsystems, pp. 419 (Elsevier, 2013). ${ }^{3}$ S. Rumley, D. Nikolova, R. Hendry, Q. Li, D. Calhoun, and K. Bergman, J. Lightwave Technol. 33(3), 547 (2015).

${ }^{4}$ S. Assefa, S. Shank, W. Green, M. Khater, E. Kiewra, C. Reinholm, S. Kamlapurkar, A. Rylyakov, C. Schow, F. Horst, H. P. Pan, T. Topuria, P. Rice, D. M. Gill, J. Rosenberg, T. Barwicz, M. Yang, J. Proesel, J. Hofrichter, B. Offrein, X. X. Gu, W. Haensch, J. Ellis-Monaghan, and Y. 
Vlasov, in 2012 IEEE International Electron Devices Meeting (IEDM) (IEEE, 2012), pp. 33.8.1-33.8.3.

${ }^{5}$ F. Boeuf, S. Cremer, E. Temporiti, M. Fere, M. Shaw, N. Vulliet, O. Bastien, D. Ristoiu, A. Farcy, T. Pinguet, A. Mekis, G. Masini, P. Sun, Y. Chi, H. Petiton, S. Jan, J.-R. Manouvrier, C. Baudot, P. Le-Maitre, J. F. Carpentier, L. Salager, M. Traldi, L. Maggi, D. Rigamonti, C. Zaccherini, C. Elemi, B. Sautreuil, and L. Verga, paper presented at the Optical Fiber Communication Conference (OSA, 2015), Paper No. W3A.1.

${ }^{6}$ See http://www.top500.org for Top500.

${ }^{7}$ L. Alloatti, S. A. Srinivasan, J. S. Orcutt, and R. J. Ram, Appl. Phys. Lett. 107(4), 41104 (2015).

${ }^{8}$ J. S. Orcutt, B. Moss, C. Sun, J. Leu, M. Georgas, J. Shainline, E. Zgraggen, H. Li, J. Sun, M. Weaver, S. Urosevic, M. Popovic, R. J. Ram, and V. Stojanovic, Opt. Express 20(11), 12222 (2012).

${ }^{9}$ L. Alloatti, M. Wade, V. Stojanovic, M. Popovic, and R. J. Ram, IET Optoelectron. 9(4), 163 (2015).

${ }^{10}$ M. T. Wade, F. Pavanello, R. Kumar, C. M. Gentry, A. Atabaki, R. Ram, V. Stojanovic, and M. A. Popovic, paper presented at the 2015 IEEE Optical Interconnects (OI) Conference.

${ }^{11}$ J. M. Shainline, J. S. Orcutt, M. T. Wade, K. Nammari, B. Moss, M. Georgas, C. Sun, R. J. Ram, V. Stojanovic, and M. A. Popovic, Opt. Lett. 38(15), 2657 (2013).
${ }^{12}$ C. Sun, M. T. Wade, Y. Lee, J. S. Orcutt, L. Alloatti, M. S. Georgas, A. S. Waterman, J. M. Shainline, R. R. Avizienis, S. Lin, B. R. Moss, R. Kumar, F. Pavanello, A. H. Atabaki, H. M. Cook, A. J. Ou, J. C. Leu, C. Yu-Hsin, K. Asanovic, R. J. Ram, M. Popovic, and V. M. Stojanovic, Nature $\mathbf{5 2 8}(7583), 534$ (2015).

${ }^{13}$ C. Sun, M. Wade, M. Georgas, S. Lin, L. Alloatti, B. Moss, R. Kumar, A. Atabaki, F. Pavanello, R. J. Ram, M. A. Popovic, and V. Stojanovic, paper presented at 2015 Symposium on VLSI Circuits Digest of Technical Papers.

${ }^{14}$ C. T. DeRose, D. C. Trotter, W. A. Zortman, A. L. Starbuck, M. Fisher, M. R. Watts, and P. S. Davids, Opt. Express 19(25), 24897 (2011).

${ }^{15}$ G. Masini, G. Capellini, J. Witzens, and C. Gunn, in 2007 th IEEE International Conference On Group IV Photonics (IEEE, 2007), pp. 28-30.

${ }^{16}$ S. Assefa, F. N. Xia, S. W. Bedell, Y. Zhang, T. Topuria, P. M. Rice, and Y. A. Vlasov, Opt. Express 18(5), 4986 (2010).

${ }^{17}$ J. Michel, J. Liu, and L. C. Kimerling, Nat. Photonics 4(8), 527 (2010).

${ }^{18}$ X. Zheng, Y. Luo, G. Li, I. Shubin, H. Thacker, J. Yao, K. Raj, J. E. Cunningham, and A. V. Krishnamoorthy, Opt. Express 20(10), 11478 (2012).

${ }^{19}$ S. Lischke, D. Knoll, C. Mai, L. Zimmermann, A. Peczek, M. Kroh, A. Trusch, E. Krune, K. Voigt, and A. Mai, Opt. Express 23(21), 27213 (2015). 